Received: December 15, 2017

Revision received: March 22, 2018

\title{
The Influence of the Different NP Word Orders in English and Chinese on E-C Translation Instruction
}

\author{
Dan Zhang ${ }^{1}$ \\ Zhejiang University
}

\begin{abstract}
In E-C translation instruction studies, the influence of the sentence length has been extensively explored by the translation theorists. Most of them come to the conclusion that the sentence average length in translational Chinese is longer than that of the original Chinese, but few of them has explained how the long sentences are constructed and why. By comparing the differences in the sentence length and the internal grammatical structures of the long sentences between original Chinese and translational Chinese, this paper will verify the fact that the difference in the orders of the noun modifiers in English and Chinese contributes to the long sentences in translational Chinese, which should be particularly instructed in E-C Translation lessons.
\end{abstract}

\section{Keywords}

Sentence Length • The Orders of The Noun Modifiers • Translational Chinese

\footnotetext{
${ }^{1}$ Correspondence to: Dan Zhang, School of International Studies, Zhejiang University, Hangzhou 310058, China. Email: newworld272@126.com
} 
In his original work on word-order typology, Greenberg (1966) noted a strong correlation between clausal word-order (O-V vs. V-O) and NP word order (Mod-N vs. N-Mod, respectively). That is:

$$
\begin{array}{lll}
\underline{\text { clausal order }} \quad \underline{\text { NP order }} \\
\mathrm{OV} \supset \text { Mod-N (pre-nominal modifiers) } \\
\text { VO } \supset \text { N-Mod (post-nominal modifiers) }
\end{array}
$$

Dryer (1988) found that these correlations are often weak, partial or unidirectional, with some languages (OV) consistent with them while others (VO) less consistent. English and Chinese present a problem for Greenberg's (1966) prediction. In Chinese, the consistent NP word-order is Mod-N which doesn't harmonizes with the VO clausal order. In English, Mod-N applies to shorter and smaller modifiers while larger modifiers such as phases and clauses conform to the VO-harmonic order N-Mod.

Due to the differences in the modifier orders in Chinese and English, most translators have to deal with the tricky problem in the translation process from English to Chinese. For readers of the translational works in Chinese from English, it can be intuitively recognized that the Chinese translational language is often odd and wordy, which is very different from the concise and smooth original Chinese.

The linguistic features in the original source language exerts essential influence on the language features in the translational language. Scholars specializing in Chinese translational language (CTL) have set up a series of norms to describe the translational language, in which the sentence length is a crucial parameter and most of them have come to the agreement that the sentence length in CTL is longer than that in the original Chinese.

In the Chinese translational language studies based on corpus, the parameter of sentence length has been extensively adopted by the translation theorists. Most of them come to the conclusion that the sentence average length is longer than that of the original Chinese, but few of them has explained how the long sentences are constructed and why.

By comparing the differences in the sentence length and the internal structures of the long sentences between original Chinese and translational Chinese, this paper will verify the fact that the difference in the orders of the noun modifiers in English and Chinese contributes to the long sentences in translational Chinese.

\section{Longer Sentence length in the translational language}

All the studies above have mentioned that the longer sentences in CTL may be affected by the different noun modifier orders between Chinese and English. However, until now, there is no analysis on the internal structures of the long sentences in translation and verifying the fact that the difference in the orders of the noun modifiers contribute to the long sentences in Chinese translational language.

In the researches based on the corpus, the sentence average length is always a prevailing perspective in analyzing the translation features because the sentence average length directly reflects the information load in a meaningful sentence which is led by the native speakers' way of thinking. It is apparent that different 
languages and different translators may lead to the difference in the sentence information load, which will further result in the difference in the sentence average length.

Since Mona Baker put forward the translation universals: simplification, explicitness and normalization, etc (Baker, 1998), large numbers of studies on these subjects have emerged, such as Kennedy (1998), Munday (1998), Laviosa (2002), Olohan (2004) and so on. They all verified Baker's hypothesis. In their studies, they analyzed the sentence length, the type token ratio, lexical density, word diversification and information load, etc, based on the synchronic corpora. They combined the theoretical explanation and experimental studies to describe the features of the translation corpora.

Baker (1993) first proposed the experimental method based on the corpus to describe the translators' stylistic features and pointed out that we must focus on the particularity of the translators' language. Based on the TEC corpus, Baker compared the language features of the two literature translators, Peter Bush and Peter Clark, by analyzing the type token ratio, sentence average length and narrative constructions in both translators' works. By firstly making a successful analysis on the stylistic features of the translation texts, Baker set up a feasible model to analyze translation texts.

Libo \& Kefei (2006) formulated a framework of corpus-based translational stylistics, analyzing and explaining the stylistic features of different types of translational texts in terms of linguistic forms and modes of literary narration. The linguistic forms include the average sentence length, the standard deviation of sentence length, type/token ratio, standardized type/token, lexical density and key word which can be calculated by WordSmith Tools. Through the analysis on the average sentence length and the standard deviation of sentence length, we can figure out the distribution of sentences in different length.

Qin (2010) also compared the average sentence length in Chinese translational novels with that in the Chinese original texts based on a corpus consisting three translation versions of Pride and Prejudice. The statistics showed that the average sentence length in the translation texts was between 23.63 27.15, which was much longer than LCMC's 16.68. Therefore, she came to the conclusion that the translators, as learners of English, were accustomed to the English long sentences and the English way of thinking, which resulted in the longer sentences in the translational works.

Numbers of corpus studies on translational language hold the belief that the sentence length calculation is an important parameter in the description of the language features. The study aims to observe the micro features of translational Chinese grammatically in order to find out the difference in sentence length between translational Chinese and original Chinese and figure out the influence of the different NP word orders in Chinese and English on the longer sentences in translational Chinese.

\section{Methodology}

The comparable data we use are from the General Chinese-English Parallel Corpus (GCEPC) created by Beijing Foreign Studies University and the English-Chinese Diachronic Corpus of the 20th century which is under construction. GCEPC has four sub-corpus, Chinese-English Literature, Chinese-English Non-Literature, English-Chinese Literature and English-Chinese Non-Literature. The Chinese texts taken from Chinese-English 
Zhang / The Influence of the Different NP Word Orders in English and Chinese on E-C Translation Instruction

and English-Chinese corpora can form comparable corpus. GCEPC enables us to take into account the English source texts in analyzing translational Chinese. The comparable corpora will be used to investigate the various translational phenomena and the differences between the original target language and the translational language.

The sentence of each length (1 30) and the sentence with "modifier+de" construction are to be extracted from comparable corpora, which can be achieved by applying the regular expressions in the data-processing tool Antconc.

With the aid of Log Likelihood Calculator, the author compares the frequencies of the sentence at each length in translational Chinese and in original Chinese.

The grammatical structures in "modifier+de" constructions are to be annotated and their frequencies will be calculated, with a computer program for annotation Anno Tool.

Table 1

Relative Frequency of the Sentence Length

\begin{tabular}{ccccccc}
\hline SSL & OCL & TCL & Loglikelihood & OCNL & TCNL & Loglikelihood \\
\hline 1 & 48.32079 & 47.8636 & 0.049819 & 30.34225 & 37.99655 & -47.0422 \\
\hline 2 & 66.07731 & 55.07481 & 76.45161 & 39.47885 & 28.34363 & 103.826 \\
\hline 3 & 98.32867 & 67.60172 & 452.5148 & 61.87201 & 39.54622 & 282.0557 \\
\hline 4 & 120.4857 & 81.91507 & 585.3709 & 74.15917 & 48.60766 & 304.4842 \\
\hline 5 & 122.9319 & 86.59904 & 501.9872 & 76.48573 & 52.22751 & 260.7869 \\
\hline 6 & 110.1945 & 81.719 & 331.6518 & 71.05708 & 53.20936 & 145.0631 \\
\hline 7 & 91.25699 & 73.026 & 157.8993 & 62.28401 & 51.97909 & 52.15137 \\
\hline 8 & 69.97166 & 62.482 & 31.90406 & 51.57213 & 49.29378 & 2.861611 \\
\hline 9 & 50.65459 & 50.303 & 0.012084 & 40.76331 & 41.29699 & -0.19234 \\
\hline 10 & 36.75 & 37.95108 & -2.04176 & 31.0693 & 36.86091 & -27.1521 \\
\hline 11 & 26.75 & 29.7595 & -13.9075 & 23.58068 & 32.10543 & -71.4099 \\
\hline 12 & 18.62 & 22.23253 & -26.4905 & 16.79487 & 25.88308 & -105.588 \\
\hline 13 & 12.65 & 17.27624 & -58.1084 & 12.8688 & 20.67807 & -99.1136 \\
\hline 14 & 8.47 & 12.57048 & -65.3035 & 9.185073 & 16.06454 & -102.097 \\
\hline 15 & 5.87 & 9.335268 & -64.1828 & 6.858511 & 12.58665 & -91.8887 \\
\hline 16 & 4.105 & 6.808101 & -54.3188 & 4.192659 & 9.806705 & -122.784 \\
\hline 17 & 2.33 & 5.359337 & -97.6849 & 3.029378 & 7.606407 & -107.558 \\
\hline 18 & 1.6589 & 3.485748 & -52.7982 & 2.011507 & 5.417938 & -85.4233 \\
\hline 19 & 1.2371 & 2.374666 & -29.5732 & 1.502571 & 3.915584 & -58.73 \\
\hline 20 & 0.8013 & 1.688409 & -26.2513 & 0.848226 & 2.981049 & -65.5398 \\
\hline 21 & 0.58301 & 1.15454 & -17.5743 & 0.678581 & 2.259446 & -46.832 \\
\hline 22 & 0.379593 & 0.762507 & -10.4383 & 0.29082 & 1.596991 & -50.9562 \\
\hline 23 & 0.295239 & 0.653578 & -11.0323 & 0.24235 & 1.206615 & -35.9892 \\
\hline 24 & 0.126531 & 0.533755 & -21.1775 & 0.169645 & 0.970024 & -31.7654 \\
\hline 25 & 0.084354 & 0.381254 & -16.0397 & 0.121175 & 0.591478 & -17.3884 \\
\hline 26 & 0.098413 & 0.272324 & -12.0991 & 0.121175 & 0.319398 & -4.87524 \\
\hline 27 & 0.05636 & 0.239645 & -7.69729 & 0.072705 & 0.224762 & -4.26822 \\
\hline 28 & 0.070295 & 0.130716 & -8.4039 & 0.04847 & 0.212932 & -5.76597 \\
\hline 29 & 0.014059 & 0.054465 & -1.99774 & 0.024235 & 0.118296 & -3.47768 \\
\hline 30 & 0.014059 & 0.076251 & -3.67625 & 0 & 0.118296 & -7.95025 \\
\hline & & & & & & \\
\hline & & & & & \\
\hline
\end{tabular}

\section{Account of the Sentence Length}

In order to figure out the difference between the translational Chinese and the original Chinese from the perspective of the sentence, the author will make a calculation of the sentence length based on the corpora in 
the General Chinese-English Parallel Corpus. With the aid of Antconc 3.2, the author extracts all sentences in each length from 1 to 30 above. The regular expression for the search in the Chinese corpora is applied to get the accurate account of the sentences.

With regular expression, the author gets the data from the four subcorpora. As the size of each corpus is different, the author transforms the data into the relative calculation (every 10000 words). Table 1 shows the SSL data of each corpus. In Table 1, OCT (726,511 words), TCL (934,811 words), OCNL (412,626 words), TCNL (845,340 words) respectively represent original Chinese literature, translational Chinese literature, original Chinese non-literature and translational Chinese non-literature.

Log Likelihood Ratio obviously shows the distinction in sentence length between the original Chinese and the translational Chinese.

Between OCT and TCT, the sentences with 1 word to 9 words in OCT is more than those of TCT. On the contrary, there are more sentences from 10 words to 30 words or above in TCT compared with those of OCT. In other words, OCT tends to contain more short sentences than TCT does and TCT contains more long sentences than OCT does.

The same is almost true to OCNT and TCNT. Except for the sentences with 1 word, OCNL contains more short sentences with 2 words to 8 words and there are more long sentences from 9 words to 30 words and the above in TCNL.

Based on the observation of the corpus data from Table 1, we can observe the general tendency of the sentence length in both translational Chinese and original Chinese. The Log Likelihood Ratio is applied to display the tendency in a more detailed way.

In Table 1, the Log Likelihood Ratios between OCL, TCL and between OCNL, TCNL are calculated by Log Likelihood Calculator designed by Jiajin, Xu. Firstly, for the ratio between OCL and TCL, from 1-word to 9-word, it is positive which means the frequency of 1-word to 9-word in OCL is higher and from 10-word to 30 -word or above, the ratio is negative which means higher frequency of 10-word to 30-word or above in TCL. The ratio between OCNL and TCNL presents the same distribution tendency, except that it is from 9-word the ratio becomes negative.

There has not been a generally recognized standard to distinguish long sentence and short sentence. Here, we take Arthur O. Hughes' view that sentences made up of 10 or less words can be identified as short sentences. The Log Likelihood Ratio between OCL and TCL becomes negative at 10-word and the ratio between OCNL and TCNL becomes negative at 9-word. Therefore, the frequency of long sentences in translational Chinese is higher than that in original Chinese, which is an important feature of translational Chinese.

The more the log likelihood ratio deviates from 0 , the more significant the difference in the frequencies of sentences in $\mathrm{OC}$ and TC at each length is. The ratios of the super-long sentences in both OC and TC are closer to 0 than the relative-long sentences, which indicate that there is not a big difference in the relative frequencies of the super-long sentences in OC and TC. According to the observation on the super-long sentences in translational Chinese, their internal structures are quite different from those in original Chinese. 
Zhang / The Influence of the Different NP Word Orders in English and Chinese on E-C Translation Instruction

Inferring from the data based on corpus, the long sentence is the significant difference between translational Chinese and original Chinese. Therefore, the features of the longer sentences in translational Chinese and original Chinese need to be investigated in detail in order to figure out the remarkable characteristics of translational Chinese and the factors that lead to its difference from original Chinese.

\section{Internal Structures of the Long Sentences in Translational Chinese}

Being left-branching and close-ending, a Chinese construction is sensitive to its load capacity, and "it resents the insertion of long modifiers" (Lian, 2006).

\section{Modifiers in Long Sentences in Translational Chinese}

In order to find out the factors that lead to the long sentences in translational Chinese, the author makes a delicate qualitative observation on the longest sentences in translational corpora with 30 or more words. In this way, the author discovers that in almost each sentence with 30 or more words, there must be more than one "modifier+de" constructions. Therefore, the author makes a hypothesis that the longer the sentence is, the more it depends on "modifier+de" construction. In order to prove the hypothesis, we need the data based on the corpora.

Table 2

The Relative Frequency of the "modifier + de" $(100 \%)$

\begin{tabular}{rcccc}
\hline SS length & OCNL & TCNL & OCL & TCL \\
\hline 1 & 0 & 0.031133 & 0 & 0 \\
\hline 2 & 4.910988 & 6.803005 & 0 & 3.401898734 \\
\hline 3 & 8.147278 & 9.871373 & 5.047183 & 7.05768611 \\
\hline 4 & 14.01961 & 16.79241 & 7.199533 & 10.35904255 \\
\hline 5 & 25.6654 & 27.42922 & 13.22049 & 17.44654088 \\
\hline 6 & 38.60846 & 38.99511 & 20.43889 & 26.89949347 \\
\hline 7 & 50.07782 & 51.04688 & 29.2867 & 36.9928401 \\
\hline 8 & 59.21053 & 62.17903 & 40.53457 & 47.36750349 \\
\hline 9 & 71.22473 & 71.92781 & 49.5698 & 57.16760502 \\
\hline 10 & 80.57722 & 81.3543 & 59.14308 & 68.91504018 \\
\hline 11 & 91.05858 & 94.25203 & 72.20179 & 81.84480234 \\
\hline 12 & 98.1241 & 105.1645 & 80.90566 & 94.41450269 \\
\hline 13 & 108.0979 & 117.1625 & 90.66667 & 105.9899117 \\
\hline 14 & 115.5673 & 127.9087 & 101.6584 & 120.9705373 \\
\hline 15 & 125.7951 & 140.3195 & 117.4641 & 131.9719953 \\
\hline 16 & 127.1676 & 152.5935 & 126.3699 & 137.76 \\
\hline 17 & 146.4 & 166.874 & 133.1325 & 158.7398374 \\
\hline 18 & 154.2169 & 171.179 & 154.2373 & 174.6875 \\
\hline 19 & 166.129 & 190.3323 & 156.8182 & 172.4770642 \\
\hline 20 & 182.8571 & 186.9048 & 171.9298 & 188.3870968 \\
\hline 21 & 189.2857 & 203.6649 & 161.5385 & 218.8679245 \\
\hline 22 & 241.6667 & 204.4444 & 188.8889 & 218.5714286 \\
\hline 23 & 160 & 233.3333 & 195.2381 & 235 \\
\hline 24 & 214.2857 & 251.2195 & 177.7778 & 246.9387755 \\
\hline 25 & 220 & 240 & 233.3333 & 240 \\
\hline 26 & 220 & 233.3333 & 200 & 268 \\
\hline 27 & 200 & 242.1053 & 200 & 309.0909091 \\
\hline 28 & 250 & 222.2222 & 300 & 291.6666667 \\
\hline 29 & 300 & 290 & 400 & 280 \\
\hline $30+$ & 142.858 & 305.128 & 250 & 300 \\
\hline & & & &
\end{tabular}


"Modifier+de" Construction in Each Corpus At first, by Antconc, the author counts the frequency of the "modifier $+d e$ " construction in each sub-corpus. Due to the different sizes of the four corpora, the relative frequency (the percentage of "modifier+de" construction in the absolute frequency of sentences at each corresponding length) is displayed here.

Table 2 can be transformed into Figure 1 and Figure 2.

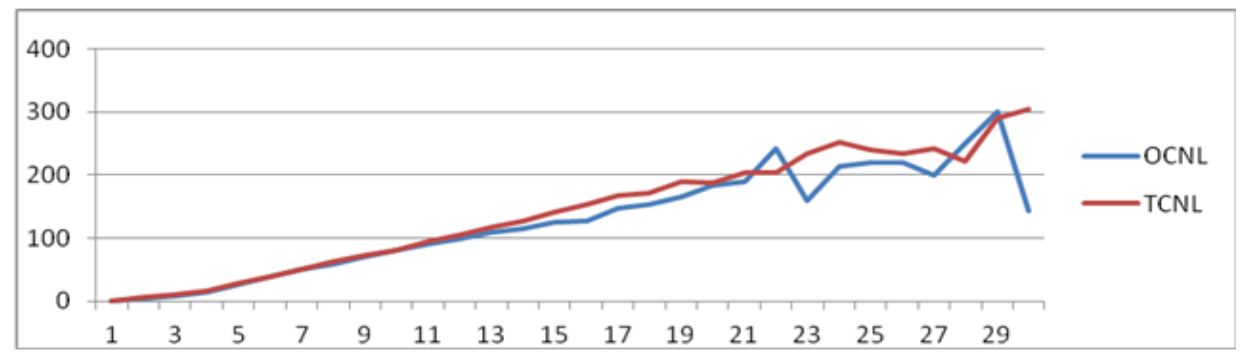

Figure 1. The Relative Frequency of "modifier+de" in non-literature corpora.

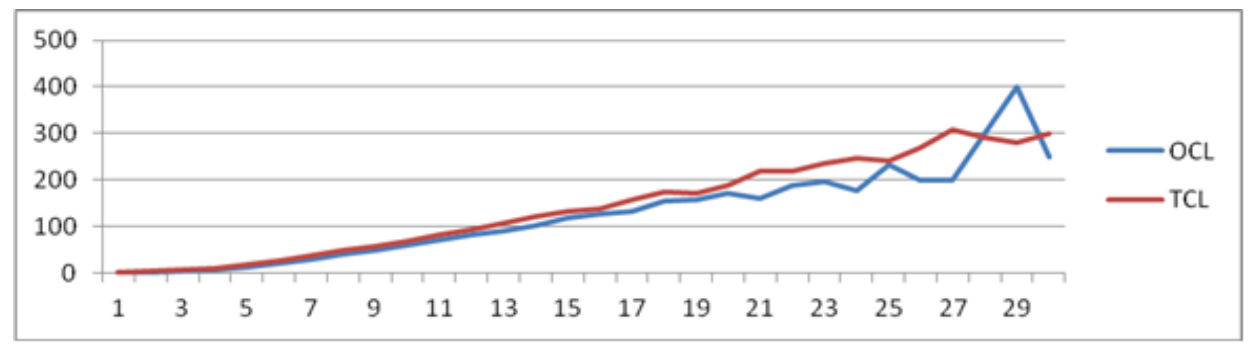

Figure 2. The Relative Frequency of "modifier+de" in literature corpora

It can be seen obviously from Figure 1 and 2 that the longer the sentence is, the more frequently the "modifier+de" is used. From sentence with 12-13 words on, the frequency is more than $100 \%$, which means that some long sentences with more than 12-13 words consist of more than one "modifier+de".

In addition, it is a general tendency that in translational Chinese, the frequency of "modifier $+d e$ " is higher than that in original Chinese, except for that at only a few super-long SS length points, the frequency in original Chinese is higher than that in translational Chinese, that is to say translational Chinese tends to contain more modifiers before the headword. For super-long sentences are rare in original Chinese, their structures to large extent may take the features of the super-long sentences in translationl Chinese, which will be analyzed in details in the following part.

Multiple "modifier $+d e$ " constructions at each sentence length One significant characteristic presented in Figure 1 and 2 is that the long sentence may contain more than one "modifier+de" constructions. Therefore, by the aid of the regular expressions, the author retrieves the sentences with two or three or more "modifier+de" construction in Antconc. 
Zhang / The Influence of the Different NP Word Orders in English and Chinese on E-C Translation Instruction

Table 3 presents the relative frequency of the sentences with two or three and above "modifier+de" constructions, which is the percentage of those sentences in their corresponding SS length.

Table 3 can be transformed into Figure 3 and Figure 4.

Table 3

Relative Frequency of More Than One "modifier+de" Constructions in Each Sentence Length

\begin{tabular}{|c|c|c|c|c|}
\hline SS Length & TCL & OCL & TCNL & OCNL \\
\hline 1 & 0 & 0 & 0 & 0 \\
\hline 2 & 0 & 0 & 0 & 0 \\
\hline 3 & 0 & 0 & 0 & 0 \\
\hline 4 & 0.013297872 & 0.023337223 & 0 & 0.032679739 \\
\hline 5 & 0.113207547 & 0.091491308 & 0.20385051 & 0.126742712 \\
\hline 6 & 0.21327646 & 0.242408778 & 0.489106269 & 0.443383356 \\
\hline 7 & 0.835322196 & 0.647049761 & 1.365498407 & 0.778210117 \\
\hline 8 & 2.440725244 & 1.567524116 & 3.335733141 & 2.255639098 \\
\hline 9 & 4.547423127 & 3.247293922 & 6.731595531 & 5.291319857 \\
\hline 10 & 8.783008037 & 5.776587605 & 9.788189987 & 9.048361934 \\
\hline 11 & 12.70131772 & 8.933263269 & 15.95431098 & 13.77183967 \\
\hline 12 & 19.45124939 & 12.37735849 & 21.023766 & 18.47041847 \\
\hline 13 & 24.71626734 & 17.66666667 & 29.29061785 & 23.16384181 \\
\hline 14 & 33.18890815 & 23.38308458 & 36.15611193 & 30.07915567 \\
\hline 15 & 40.02333722 & 29.66507177 & 43.13909774 & 31.09540636 \\
\hline 16 & 41.76 & 34.5890411 & 49.45717732 & 32.94797688 \\
\hline 17 & 55.89430894 & 38.55421687 & 57.07620529 & 41.6 \\
\hline 18 & 58.125 & 52.54237288 & 60.26200873 & 45.78313253 \\
\hline 19 & 56.88073394 & 51.13636364 & 66.4652568 & 51.61290323 \\
\hline 20 & 65.80645161 & 54.38596491 & 63.88888889 & 60 \\
\hline 21 & 72.64150943 & 56.41025641 & 75.39267016 & 60.71428571 \\
\hline 22 & 74.28571429 & 70.37037037 & 65.92592593 & 66.66666667 \\
\hline 23 & 76.66666667 & 66.66666667 & 79.41176471 & 50 \\
\hline 24 & 81.63265306 & 55.55555556 & 84.14634146 & 85.71428571 \\
\hline 25 & 68.57142857 & 100 & 80 & 80 \\
\hline 26 & 92 & 71.42857143 & 74.07407407 & 80 \\
\hline 27 & 90.90909091 & 75 & 73.68421053 & 66.66666667 \\
\hline 28 & 91.66666667 & 80 & 77.77777778 & 100 \\
\hline 29 & 100 & 100 & 90 & 100 \\
\hline $30+$ & 86.66666667 & 100 & 91.66666667 & 60 \\
\hline
\end{tabular}

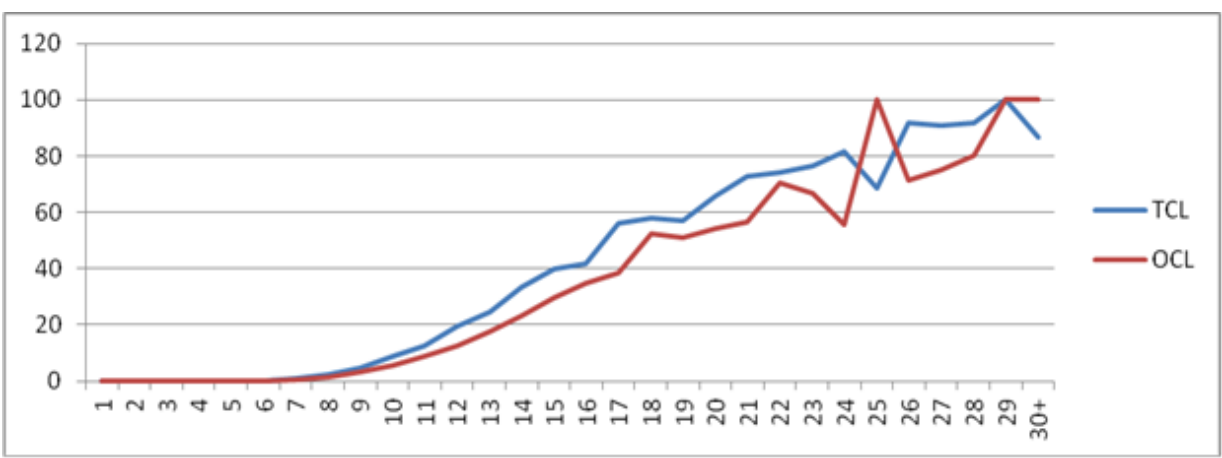

Figure 3. Relative frequency of more than one "modifier+de" constructions in each sentence length. 


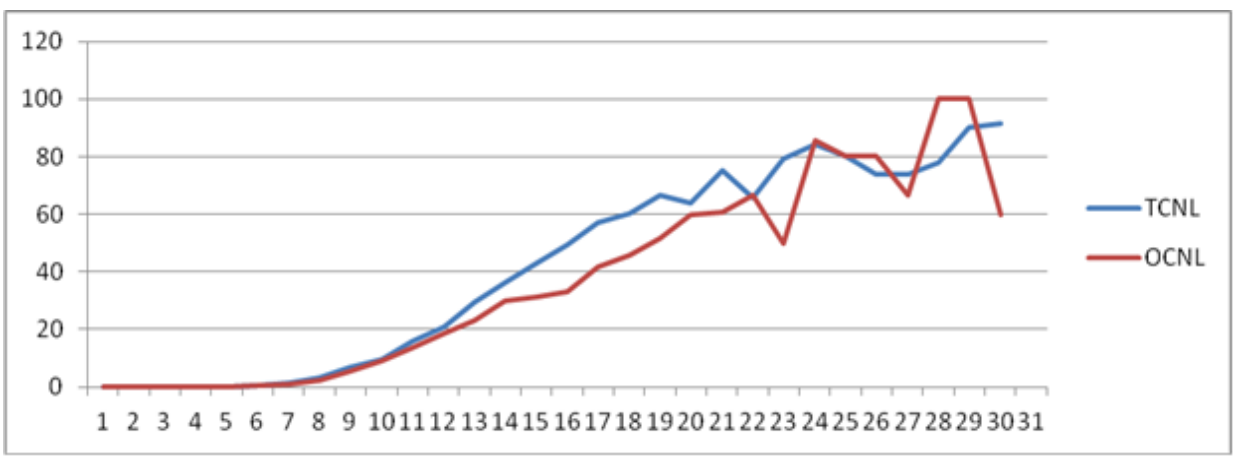

Figure 4. Relative frequency of more than one "modifier+de" constructions in each sentence length.

From Table 3 and Figure 3, Figure 4, we can see that the percentage of the sentences with two or three or more "modifier+de" construction increases as the SS length increases. We can infer that the longer the sentence is, the more dependent it is on the "modifier+de" construction. In other words, "modifier+de" plays a crucial role in the generation of the long sentences. Particularly, the super-long sentences rely heavily on the two and three or more "modifier+de" construction. Besides, the percentage of sentences with two or three and above "modifier+de" constructions in translational Chinese tends to be higher than that in original Chinese. There are a few exceptions at the super-long SS length, which result from the small amount of the super-long sentence in original Chinese. However, the exceptions won't affect the overall tendency. The higher percentage of two and three "modifier+de" is an important feature of translational Chinese.

\section{Grammatical Analysis}

Generally speaking, it is necessary to conduct both the semantic and grammatical analysis for a comprehensive investigation on the sentence structure. The author observes all the phrases of "three 'modifier+de' constructions +headword" and classified them into two categories, which are marked as "embedded modifier" and "non-embedded modifier" with the aid of Anno Tool.

The Increase of embedded constructions Firstly, the frequency of "three 'modifier+de' constructions +headword" in translational Chinese is much higher than that in original Chinese. The phrases with three or more modifiers in Chinese are rare, while they are very common in translational Chinese. Secondly, the proportion of the embedded modifier in translational Chinese is higher than that of the non-embedded modifiers. The embedded modifiers impose a huge burden on the readers' interpretation of the translational Chinese, which are to large extent not acceptable in original Chinese. The following problematic instances with embedded modifiers can clarify the negative effects of embedded modifiers.

So far, we have got an overall calculation of the embedded modifiers in the translational Chinese and their negative effects on the translational language. On contrary, the frequency of the embedded modifiers in original 
Chinese is rather low. Occasionally, there are several embedded modifiers in original Chinese, but their constructions are very simple and their meaning is easy for readers to grasp.

Table 4

\begin{tabular}{lcccc} 
The Frequency of the "embedded modifier" and "non-embedded modifier" \\
\hline Type & TCL & OCL & TCNL & OCNL \\
\hline embedded modifier & 131 & 10 & 93 & 8 \\
non-embedded modifier & 100 & 14 & 72 & 17 \\
\hline
\end{tabular}

Internal Structure of the Sentences with three or more "modifier+de" constructions: The modifiers before the headwords can fall into a series of grammatical categories, and the most common categories are adjectives, nouns, as well as pronouns, verbs, and so on. In order to describe the grammatical structure features of "modifier+de" in translational Chinese sentences, which are to be compared with those of original Chinese, the author also uses Anno Tool to annotate the sentences with three or mores in translational Chinese and original Chinese.

With the help of Anno Tool, the author annotates every component of the modifiers before "de" with its grammatical category. To sum up, the annotated components before "de" can be classified into three types: word, phrase and clause.

By Antconc, the author calculates the frequency of the components of the modifiers in the sentences with three or more "modifier+de" constructions in translational Chinese and original Chinese. The following Figure 5, Figure 6 and Figure 7, presenting respectively the contrast in the relative frequency of words, phrases and clauses in the modifiers between translational Chinese and original Chinese.

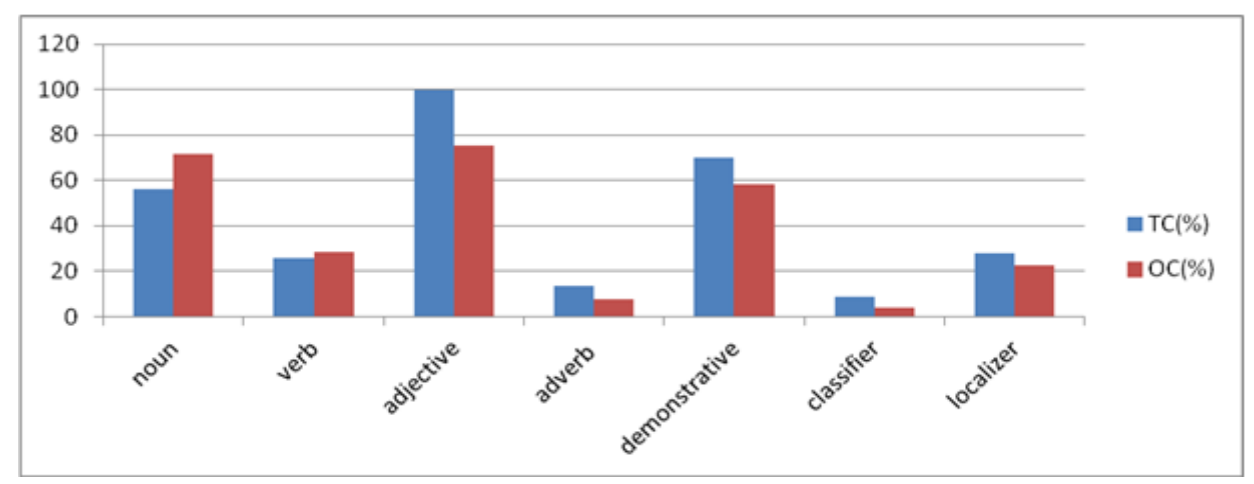

Figure 5. Words.

In the Figure 5 for words in the modifiers, we can see that in translational Chinese, the frequencies of the content words -- nouns and verbs are lower than those in original Chinese while the frequencies of the functional words - demonstratives, classifiers and localizers are higher, which can be attributed to the influence of the higher frequencies of functional words in English on the target language - Chinese. This result is consistent with the research by Hongwu Qin and Kefei Wang (2009).

We have mentioned that in the translation from English to Chinese, the modifiers positioned on the right side of the headwords are always transformed onto the left side in Chinese, the prepositional phrases in English tend to be placed before the headwords in Chinese, so the localizers before "de" in translational Chinese occurs 
more frequently than those in original Chinese. In addition to its independent usage before "de", the localizers are often put together with the verbs, verbal phrases and the adjectives.

However, it is not the case for the content words - adjectives and adverbs whose frequencies in translational Chinese are nevertheless higher, which is contrary to our experience that the frequencies of adjectives and adverbs should be in positive correlation with the frequencies of nouns and verbs.

As the adverbs can modify both adjectives and verbs, the higher frequency of adjectives is the key contributor to the higher frequency of adverbs. The function of adverbs is to modify adjectives and verbs, so the greater number of the adjectives and the phrases with verbs contribute to the higher frequency of the adverbs in translational Chinese.

In terms of the frequency of pronouns, it has been proved (Huang, 2008) that in translational Chinese, the personal pronouns are more explicit than those in original Chinese. The pronouns in translational Chinese are influenced by English and are more frequently used, for English discourse achieves the cohesion which relies to large extent on the use of pronouns. He Zhaoxiong (1986) pointed out that in English, anaphora of the pronoun is normal and unmarked while in Chinese, zero anaphora is normal and unmarked. Hu Zhuanglin (1996) also proved that the zero anaphora in Chinese is much more than those in English and the zero anaphora is not influenced by syntax for Chinese is of parataxis.

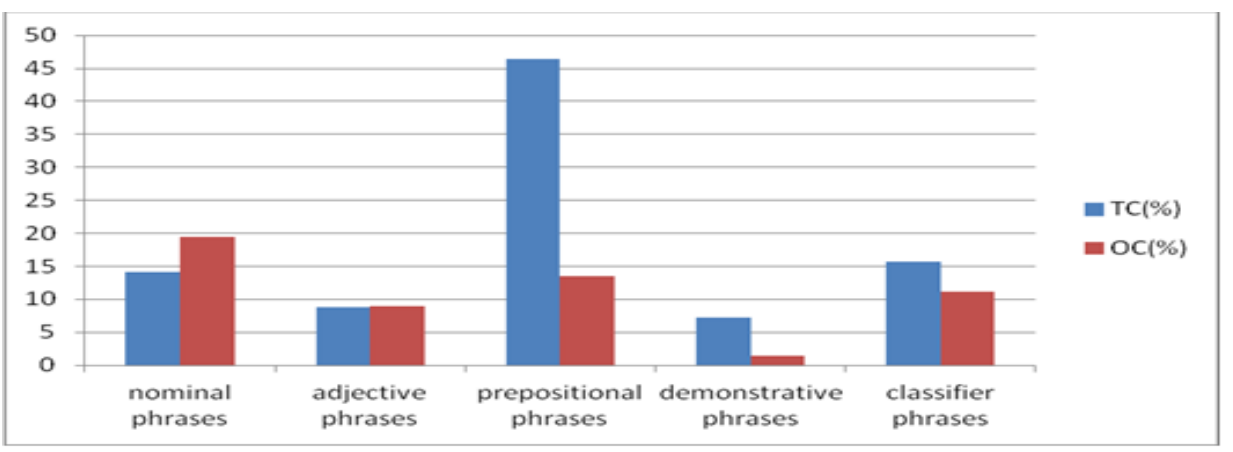

\section{Figure 6. Phrases.}

According to the statistics in Figure 6, the frequencies of verbal phrases, prepositional phrases, pronoun phrases and quantitative phrases are higher in translational Chinese than those in original Chinese. On the contrary, in original Chinese, the frequencies of these four phrases in modifiers are much lower and in most cases, they are in their much more simple form which will not impact the fluency and brevity of the discourse.

We can see that the prepositional phrase is a closed construction which can be extended by various modifier constructions without limitation. However, in original Chinese, the complicated modifiers embedded in the prepositional phrases are very rare and most of the modifiers in the prepositional phrases are in the simple form like the first instance above. If the modifiers in the prepositional phrases are long and complex, the distance between the preposition and the word at the end of the phrase is too long so that the readers have to reread to get a comprehensive understanding for they have been distracted by the overloaded modifier information. 
Now let's see what is peculiar to the demonstrative phrases in translational Chinese. The demonstrative phrases are phrases headed by a demonstrative word and ended with the headword referred to by this word.

\section{$\mathrm{Eg}$ : 这个 既像她的慈爱的对象 又像她的罪蒠和折磨的标志 的 作品}

This pronoun phrase is filled by two "copular-predicative" clauses as the modifiers for the headword “作品 ” connected by the conjunctions “既...... 又........ In the modifiers, “她的慈爱的对象” and “她的罪酕和折 磨的标志” are the predicative for each copular “像”. In addition, one predicative is modified by the pronoun “ 她” and the adjective “慈爱” and the other predicative is modified by “她” and the nouns “罪莒和折磨” linked by a conjunction. All the information of modification is inserted between the pronoun "这个” and the headword “作品” and probably the readers can't bear all of them in mind to go on with the discourse so that they may return in order to go over the information loaded here when they need.

So far, we have analyzed the phrasal components that occur obviously more frequently in the modifiers of translational Chinese and found that it is the great frequency of modifiers and their complicated structure that contribute to the extended load capacity of these phrases. On the contrary, in original Chinese, the frequencies of these four phrases in modifiers are much lower and, in most cases, they are in their much simpler form which will not impact the fluency and brevity of the discourse.

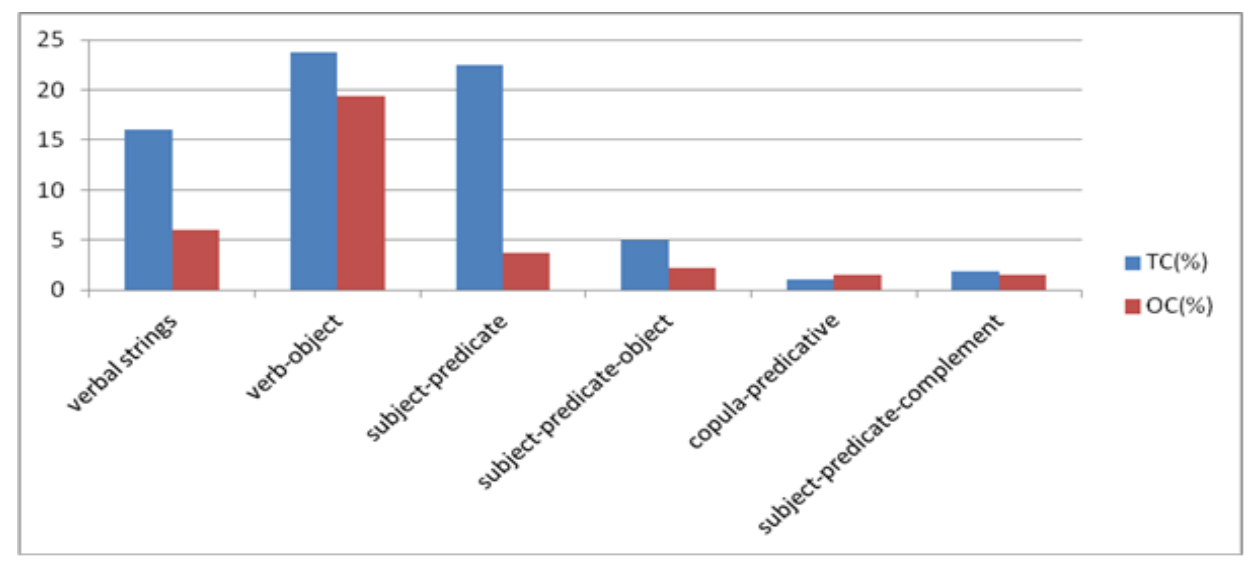

Figure 7. Clause.

In Figure 7, the frequency of the clausal components of the modifier is presented. We can see that the modifiers at the clausal level in translational Chinese are much more than those in original Chinese. It is certain that the clausal modifiers will lead to the complexity of translational Chinese. Although there are also clausal modifiers in original Chinese, most of them will not impact the readability of the discourse because there are not redundant embedded components in these clausal modifiers.

Verbal-Strings:

The use of non-finite verbs and the subordinate clauses before or after the main sentence in English can lead to the extension of the sentence length in translational Chinese (Wang,1989). The past participle phrases, the present participle phrases, the infinitive phrases or the subordinate clauses are always transformed into the 
"modifier+de" construction before the headwords. The verbal strings defined in translational Chinese are the strings made up by verbs in fixed constructions or in common expressions. Besides, some phrases with verbs that are closely related or are difficult to be exactly classified as any kind of phrases are also defined as verbal strings.

$\mathrm{Eg}$ : 把 矿砂 运上 苏必利尔湖双港 的货船

“把......verb......”is a fixed construction in Chinese, in which the word behind “把” is the object and the part behind the second verb is the adverbial. In the instance, “矿砂” is the object and “运上 苏必利尔湖双港 的货船” is the verb and its adverbial. The constructions like “将......verb.....”, “使......verb.....” and “让 ......verb......” are all of this kind. “将道德生活的光束传送给 孕育着的胎儿的媒介”一 this is an example for “将......verb......" In this kind of construction, the object and the adverbial can be modified by various forms mentioned in last section, so the load capacity of this construction may be so extended that the readability becomes unsatisfying.

Verb-object:

$\mathrm{Eg}$ : 佩戴 这个光彩动人的丝绣徽记 的人

“人” is modified by the clausal modifier “佩戴 这个光彩动人的丝绣徽记” in verb-object form in which the object “丝绣徽记” is modified by the adjective phrases “光彩动人” headed by the pronoun “这个”.

The "verb-object" modifiers in translational Chinese often embed in other modifiers or lie in parallel with other modifiers before "de". However, in original Chinese, not only the frequency of the "verb-object" modifiers is lower, but also the form is usually rather simple.

Subject-predicate:

$\mathrm{Eg}$ : 作为对 他所落入或正要落入的幻觉的一种无声的 对抗。One of modifiers for the headword “本 分” is “爸爸伤心而体贴地指出” which is a subject-predicate form. However, its predicate is modified by two adverbs “伤心” and “体贴” connected by the conjunction.

In the second example, in the embedded modifier “他所落入或正要落入” for the headword “幻觉”, “他” is the subject and the predicates are in parallel modified by some trivial adverbs.

In a word, the subjects and predicates in most of the "subject-predicate" modifiers in translational Chinese are always modified by other modifiers while in original Chinese the "subject-predicate" modifiers are in their simple forms.

Subject-predicate-object:

$\mathrm{Eg}$ : 他所选择的这个英国家庭所带给他的 幸福 和 永远积极工作的 需要。

“幸福” is the headword modified by the modifier “他所选择的 这个英国家庭所带给他” which is in the form of subject-predicate-object and the subject is modified by the other "subject-predicate" modifier “他所选 择”. 
In translational Chinese, the clausal modifiers are always embedded by other modifiers modifying the elements in them and the embedded modifiers can be either simple or complex. For original Chinese, there are few modifiers in such a complicated form and even if this kind of modifiers is present, they are in their simplest form.

\section{Discussion and Conclusion}

The internal grammatical structure of the modifiers in translational Chinese is different from that of original Chinese. In translational Chinese, for the category of words, the frequencies of the functional words, the adjectives and the adverbs are higher than those in original Chinese. In terms of phrases, except for that the frequency of the adjective phrases are only a little bit higher than that in original Chinese, the frequencies of demonstrative phrases, prepositional phrases and classifier phrases in closed-end construction are higher. As for the clausal modifiers, almost all kinds of clausal modifiers occur more frequently than those in original Chinese, except the clausal modifiers in the structure of copula-predicative whose frequency is a little bit lower than that in original Chinese. From the data and analysis above, we can draw a conclusion that the long, complicated and embedded modifiers are peculiar to translational Chinese, which is the main factor that contributes to higher frequency of the long sentences in translational Chinese.

The author finds that the proportion of the embedded attributives in translational Chinese is higher than that of the non-embedded attributives. Bases on the analysis of the components of the attributives — words, phrases and clauses, the higher frequencies of the functional words, the closed-end constructions and almost all kinds of clausal attributives are peculiar to translational Chinese and their syntactic combination makes up of the great numbers of the long and complicated attributives in translational Chinese. The large proportion of the embedded attributives in translation Chinese stems from the post-positioned modifiers (Rel/PP/N-Comp) because the translators tend to transfer the original English structures into the Chinese typical noun structures without the consideration of the distance between the modifiers and the head nouns.

Through comparison, the awkwardness and poor readability of translational Chinese ascribe to the difference in the noun modifier orders between English and Chinese.

The rigid order of modifiers in English is as follows:

$$
\mathrm{NP}=(\mathrm{Quant})(\mathrm{Det})\left(\mathrm{AP}^{*}\right)\left(\mathrm{N}^{*}\right) \mathrm{N}(\mathrm{Pl})(\mathrm{Rel} / \mathrm{PP} / \mathrm{N}-\mathrm{Comp})
$$

It states that modifiers that precede the head noun are, in order, quantifiers (Quant), determiners (Det), adjectival phrases (AP) and modifying nouns (N). Modifiers that follow the head noun are the plural morpheme $(\mathrm{Pl})$, relative clauses (Rel), prepositional phrases $(\mathrm{PP})$ or noun complements (N-Comp).

The parentheses ( ) around a constituent indicate optional-conjunctive-choice, in the given order. The slashes / indicate exclusive - disjunctive - choice of only one of the bracketed items. The asterisk stands for optional recursion, as in the case of modifying nouns $\left(\mathrm{N}^{*}\right)$ or adjectives $\left(\mathrm{AP}^{*}\right)$.

However, in Chinese, all the modifiers for nouns are pre-positioned before the headwords, which is illustrated in the following: 
[我] [那件] [昨天下午买的] [很厚的] [棉麻] 衬衫

My that bought yesterdayafternoon very thick cotton shirt

My thick cotton shirt bought yesterday afternoon

In original Chinese, the complex and long modifiers occurs less frequently than those in translational Chinese because the native Chinese speakers always employ the run-on sentences to avoid the complex modifiers, in which way the modifiers may be scattered around the head nouns according to the semantic features. However, in order to conform to the word order of English, the translators always ignore to obey the pattern of Chinese noun modifier, which contributes to the poor readability of the noun phrases in translational Chinese. The complex and long modifiers assert massive burden for the memory. Many scholars proposed that the closer the modifiers to the head nouns, the better (Lu, 1986; Hawkins, 1994; Tang, 2006), which is the opposite situation in translational Chinese.

In a word, influenced by the order of English noun modifiers, in E-C translation lessons, the structures of the sentence are always complicated by the phrasal or clausal modifiers that are long and complex in grammatical structure, which is the primary factor that contributes to the awkwardness and poor readability of translational Chinese in E-C translation instruction.

\section{References}

Baker, M. (1992). In other words: A course book on translation. London: Routledge.

Baker, M. (1993). Corpus linguistics and translation studies: Implications and applications. Text and technology: In honour of John Sinclair, 233, 250.

Baker, M. (1998). Routledge encyclopedia of translation studies. London: Routledge.

Barrett, L., Greenberg, D. F., \& Schwartz, M. (2006). A syntactic feature counting method for selecting machine translation training corpora. Language \& Computers, 1-19.

Biber, D. (1998). Corpus linguistics. Cambridge: Cambridge University Press.

Chen, W. (2007). Translation of English corpus and descriptive translation studies based on translation English corpus. Foreign Languages: Journal of Shanghai International Studies University, (1), 67-73.

Ding, J. (2011). A corpus-based study of the two Chinese translations of the bridges of Madison county. Journal of Tianjin Foreign Studies University, 18(1), 44-49.

Ding, S. D. (2001). A brief discussion on western translation corpus research. Foreign Languages, (5), 61-66.

Dryer, M. S. (1988). Object-verb order and adjective-noun order: dispelling a myth. Lingua, 74(2-3), 185-217. http://dx.doi. org/10.1016/0024-3841(88)90059-9

Goldberg, A. E. (1994). Constructions: A construction approach to argument structure. Chicago: The University of Chicago Press.

Greenberg, J. (1966). Some universals of grammar with particular reference to the order of meaningful elements. In J. Greenberg (Eds). Language Universals, 73-113.

Hu, X. Y. (2004). Corpus translation research and translation universality. Shanghai Translation, (4), 47-49. 
Hu, X. Y., \& Zeng, J. (2009). Using corpus to investigate the capacity and structure of Chinese translation novels. Journal of PLA University of Foreign Languages, 32(3), 61-66.

Libo, H., \& Kefei, W. (2006). Reflection on translation universality research. Chinese Translation, (5), 36-40.

Hawkins, J. A. (1994). A performance theory of order and constituency. Cambridge: Cambridge University Press, http://dx.doi. org/10.1017/CBO9780511554285

Ke, F. (2003). The characteristics, distribution and English translation of Chinese "ba" sentence. Foreign Language and Foreign Language Teaching, (12), 1-5.

Ke, F. (2005). Implicit and explicit in translation. Foreign Language Teaching and Research, 37(04), 64-68.

Kennedy, G. (1998). An introduction to corpus linguistics. London: Longman.

Kubler, C. C. (1985). A study of Europeanised grammar in modern written Chinese. Taipei: Students Book Co.

Laviosa, S. (2002). Corpus based translation studies: Theory, findings, applications. New York: Rodopi.

Lian, S. N. (1993). Contrastive studies of English and Chinese. Higher Education Press.

Liu, M. Q. (1992). A contrastive study of Chinese and English sentence expansion mechanisms. Modern Foreign Languages, (1), 10-15.

Liu, M. Q. (2006). New Chinese-English contrast and translation. Chinese Foreign Translation Publishing Company.

Lu, B. F. (1986). Synchronous block process of sentence interpretation and its quantitative description. Chinese Language, (2).

Lv. S. X. (1942). Chinese grammar. Beijing: The Commercial Press.

Lv. S. X. (1984). Proceedings of Chinese grammar. Beijing: The Commercial Press.

Lv. S. X. (1999). Eight hundred words in modern Chinese. Beijing: The Commercial Press.

Olohan, M. (2004). Introducing corpora in translation studies. London: Routledge.

Parsons, T. (1990). Events in the semantics of English: a study in subatomatic semantics. Cambridge: MIT Press, http://dx.doi. org/10.1111/j.1468-0017.1993.tb00295.x

Peyraube, A. (2002). Westernization of Chinese grammar in the 20th century: Myth or reality. Journal of Chinese Linguistics, 28, 1-26.

Qin, H. W. (2010). The structural capacity of English-Chinese translation language: based on the study of multitranslation corpus. Foreign Languages Journal of Shanghai International Studies University, (4), 73-80.

Qin, H. W. (2000). Syntactic alienation and domestication in translation. Foreign Language Teaching and Research, (5), 368-373.

Qin, H. W., \& Wang, K. F. (2007). Application of corresponding corpus in translation teaching: Theoretical Basis and Implementation Principles. Chinese Translation, (5), 49-52.

Qin, H. W., \& Wang, K. F. (2004). Corpus-based translation language analysis_- Taking the Chinese corresponding structure of "so...that" as an example. Modern Foreign Language, 27(01), 44-52, 109-110.

Rothstein, S. (Ed.). (1998). Events and gramma. Netherlands: Kluwer Academic Publishers.

Stubbs, M. (2004). Language corpora. In A. Davies and C. Elder (Eds.). The handbook of applied linguistics, 106-132.

Shi, D. X., \& Zhu Z. Y. (1999). The influence of English on Hong Kong written Chinese syntax —— language changes caused by Language contact. Foreign Language: Journal of Shanghai International Studies University, (4), 2-11. 


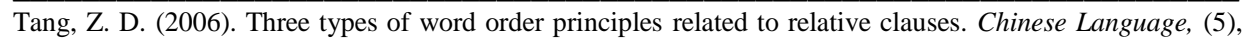
409-422.

Toury, G. (1995). Descriptive translation studies and beyond. Philadelphia: John Benjamins.

Wang, K., \& Qin, H. (2014). What is peculiar to translational mandarin Chinese? a corpus-based study of chinese constructions' load capacity. Corpus Linguistics \& Linguistic Theory, 10(1), 57-77. http://dx.doi. org/10.1515/cllt-2013-0020

Wang, K, F., \& Huang, L. B. (2007). Several terms of corpus translation studies. Journal of Sichuan International Studies University, 23(6), 101-105.

Wang, K, F., \& Qin, H. W. (2009). Discussion on the linguistic features of English-Chinese translationbased on macro-analysis of corresponding corpus. Foreign Language Journal, (1), 101-105.

Wang, K, F. (2003). A corpus survey of English-Chinese/Chinese-English sentences. Foreign Language Teaching and Research, 35(06), 11-17,82.

Xie, Y. J. (1989). Introduction to modern Chinese Europeanization grammar. HongKong, Guangming Book Company. 